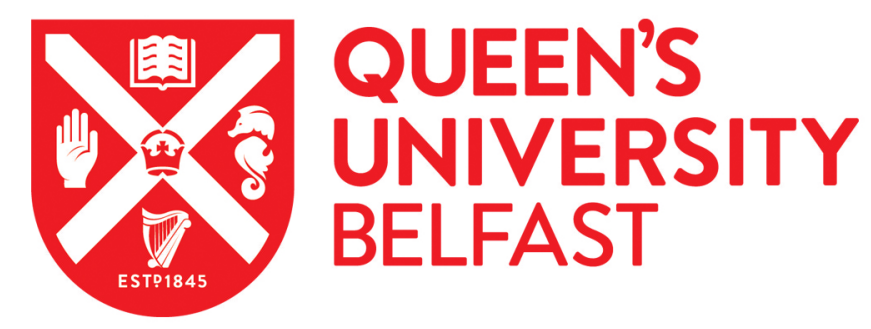

\title{
Analysis of different planar antenna arrays for mmWave massive MIMO systems
}

Tan, W., Assimonis, S. D., Matthaiou, M., Han, Y., Jin, S., \& Li, X. (2017). Analysis of different planar antenna arrays for mmWave massive MIMO systems. In IEEE Vehicular Technology Conference (VTC) Institute of Electrical and Electronics Engineers Inc.. https://doi.org/10.1109/VTCSpring.2017.8108586

Published in:
IEEE Vehicular Technology Conference (VTC)

Document Version:

Peer reviewed version

Queen's University Belfast - Research Portal:

Link to publication record in Queen's University Belfast Research Portal

Publisher rights

(c) 2017 IEEE.

This work is made available online in accordance with the publisher's policies. Please refer to any applicable terms of use of the publisher.

\section{General rights}

Copyright for the publications made accessible via the Queen's University Belfast Research Portal is retained by the author(s) and / or other copyright owners and it is a condition of accessing these publications that users recognise and abide by the legal requirements associated with these rights.

Take down policy

The Research Portal is Queen's institutional repository that provides access to Queen's research output. Every effort has been made to ensure that content in the Research Portal does not infringe any person's rights, or applicable UK laws. If you discover content in the Research Portal that you believe breaches copyright or violates any law, please contact openaccess@qub.ac.uk. 


\title{
Analysis of Different Planar Antenna Arrays for mmWave Massive MIMO Systems
}

\author{
Weiqiang Tan $\ddagger$, Stylianos D. Assimonis $\dagger$, Michail Matthaiou ${ }^{\dagger}$, Yu Han $\ddagger$, Xiao Li $\ddagger$, and Shi Jin $\ddagger$ \\ ${ }_{\ddagger}^{\ddagger}$ National Mobile Communications Research Laboratory, Southeast University, Nanjing 210096, P. R. China \\ ${ }^{\dagger}$ School of Electronics, Electrical Engineering and Computer Science, Queen's University Belfast, BT3 9DT, U. K. \\ E-mail: ${ }^{\ddagger}\{$ wqtan, hanyu, li_xiao, jinshi $\} @$ seu.edu.cn, ${ }^{\dagger}\{$ s.assimonis, m.matthaiou $\} @$ qub.ac.uk
}

\begin{abstract}
In order to reap the full scale of benefits of millimeter wave (mmWave) massive multiple-input multiple-output (MIMO) systems, the design of antenna arrays at the transmitter or receiver becomes more critical due to the propagation characteristic at $\mathbf{m m}$-frequencies. In this paper, we investigate the steering vector and array factor by considering three types of planar antenna arrays, namely uniform rectangular planar array (URPA), uniform hexagonal planar array (UHPA), and uniform circular planar array (UCPA). Based on these results, we investigate the array directivity/gain and the achievable spectral efficiency in a 3-dimensional massive MIMO system by considering both the azimuth and elevation dimensions. An important observation is that the the maximum array gain, the beamwidth, and the achievable spectral efficiency (SE) for the above three types of planar antenna array configurations are almost identical. The side lobe level and the geometric area of the UHPA configuration are systematically better than those of the UCPA and URPA configurations.
\end{abstract}

Index Terms-Achievable ergodic SE, array antenna, array gain, mmWave MIMO, planar antenna arrays.

\section{INTRODUCTION}

Massive multi-input-multi-output (MIMO), where a base station (BS) is equipped with a large number of antennas has emerged as one of the promising technologies for fifth generation $(5 \mathrm{G})$ communications since it can provide substantially higher SE and energy efficiency [1,2]. One of the major challenges is how to pack a large number of antenna elements within a finite volume, especially for the traditional 1-dimensional antenna arrays, such as the uniform linear array (ULA) and uniform circular array (UCA). For example, when 100 antenna elements are uniformly spaced with a half wavelength separation distance at a carrier frequency of $2 \mathrm{GHz}$, the length of a ULA will be about $7.5 \mathrm{~m}$. For a UCA configuration, the diameter of the circle array is about $2.4 \mathrm{~m}$. Thus, this prohibitive antenna dimensions require the development of alternative configurations.

Toward this direction, there are two feasible solutions to deploy massive MIMO antennas at the BS. The first solution is the use of mmWave carrier frequencies $(30 \mathrm{GHz}$ and beyond) since the operating wavelength becomes very small (i.e., less than $10 \mathrm{~mm}$ ), which makes it possible to pack more antenna elements within a compact physical space. In [3], the synergy between millimeter wave and massive MIMO system was proposed, which alleviates the array size constraints and improves significantly the achievable sum rate. The second solution is to use of a 2-dimensional (2D) antenna arrays since which can [4]: (a) deploy higher numbers of antenna elements at the BS site, (b) achieve more directed transmissions (i.e., narrow beam radiation pattern), and (c) provide higher antenna gains to overcome path loss. For example, the work in $[5,6]$ investigated the effect of several antenna array configurations, such as ULA, uniform planar array (UPA) and UCA, on the achievable SE and spatial fading correlation, and indicated that the antenna array configurations has an important impact on MIMO performance. As massive mmWave MIMO is a newborn technology, further studies on the different geometrical shapes of planar antenna array are of special interest.

The primary goal of this paper present the radiation characteristics of some benchmark 2D planar antenna arrays, in terms of steering vectors, array factor the directivity/gain function, and then assess how these characteristics affect the performance of mmWave massive MIMO systems. We adopt the mmWave channel model introduced in [7-9]. The radiation characteristics in terms of steering vectors, antenna array factor and directivity of three benchmark 2D planar arrays (i.e., URPA, UHPA and UCPA) is studied, and then we derive the upper bound on the achievable SE and present the achievable SE via numerical simulation for the proposed 2D planar antenna arrays, which based on a practical mmWave MIMO channel model. Interestingly, results show that the radiation characteristics and the maximum achievable SE are almost identical for all the three antenna arrays. However, in terms of side lobe level and physical space the UHPA antenna array is performs the best.

\section{SySTEM MOdEL}

In this section, we consider a point-to-point mmWave MIMO channel model and present the steering vectors and the array factor for three types of planar antenna arrays (i.e. URPA, UHPA, and UCPA).

\section{A. Channel Model}

Consider a mmWave massive MIMO system with $N_{t}$ antennas at the transmitter and $N_{r}$ antennas at the receiver. The received signal can be expressed as

$$
\mathbf{y}=\sqrt{\rho} \mathbf{H} \mathbf{x}+\mathbf{n},
$$

where $\rho$ denotes the transmitter power, $\mathbf{y} \in \mathbb{C}^{N_{r} \times 1}$ is the received signal, $\mathbf{H} \in \mathbb{C}^{N_{r} \times N_{t}}$ is the channel matrix, $\mathbf{x} \in \mathbb{C}^{N_{t} \times 1}$ 



Fig. 1. An illustration of three types of 2D planar antenna array configurations, (a) URPA, (b) UHPA, (c) UCPA.

is the transmitted signal, and $\mathbf{n} \in \mathbb{C}^{N_{r} \times 1}$ is the independent and identically distributed zero-mean additive Gaussian noise. ${ }^{1}$

For mmWave wireless propagation, the clustered geometric channel model is adopted, where the matrix channel $\mathbf{H}$ is assumed to be a sum of $P$ scattering clusters, each of which constitutes of $L$ propagation paths. Therefore, the channel matrix $\mathbf{H}$ can be parameterized as [7-9]

$$
\mathbf{H}=\gamma \sum_{p=1}^{P} \sum_{l=1}^{L} a_{p l} \Lambda_{r}\left(\phi_{p l}^{r}, \theta_{p l}^{r}\right) \Lambda_{t}\left(\phi_{p l}^{t}, \theta_{p l}^{t}\right) \mathbf{a}_{r}\left(\phi_{p l}^{r}, \theta_{p l}^{r}\right) \mathbf{a}_{t}^{H}\left(\phi_{p l}^{t}, \theta_{p l}^{t}\right),
$$

where $\gamma=\sqrt{1 / P L}$ is a normalization factor, $a_{p l}$ is the complex gain of the each path, whose entries are complex circular symmetric Gaussian random variables with zero mean and unit variance, $\theta_{p l}$ and $\phi_{p l}$ are the azimuth and elevation angle of arrival or departure of the $l$-th path at the $p$-th cluster, respectively, $\Lambda_{r}\left(\phi_{p l}^{r}, \theta_{p l}^{r}\right)$ and $\Lambda_{t}\left(\phi_{p l}^{t}, \theta_{p l}^{t}\right)$ represent the antenna element gain for the transmitter and receiver, respectively, while $\mathbf{a}_{r}\left(\phi_{p l}^{r}, \theta_{p l}^{r}\right)$ and $\mathbf{a}_{t}^{H}\left(\phi_{p l}^{t}, \theta_{p l}^{t}\right)$ represent the steering vector of the receiver and transmitter antenna array, respectively. We assume that the antenna elements are isotropic elements and there is no inter-element coupling/interference then for the gain functions, we have $\Lambda\left(\phi_{p l}, \theta_{p l}\right)=\Lambda\left(\phi_{p l}, \theta_{p l}\right)=1$.

The isotropic elements could be replaced by other antenna types such as linear antennas, patch antennas, etc., taking into account the corresponding gain functions.

Yet, it is known that the antenna array gain is proportional to the squared absolute value of the array factor. Mathematically speaking,

$$
G(\phi, \theta)=\sigma|A F(\phi, \theta)|^{2},
$$

where $A F(\phi, \theta)$ denotes the antenna array gain and $A F(\phi, \theta)$ denotes the antenna array factor, which will also be discussed in the following section. From (3), we observe that the antenna array gain depends on the array factor, which itself depends on the antenna array configuration and the excitation [11].

\section{B. Array Steering Vector}

In this section, we first present the steering vector and the antenna array factor with respect to different antenna array configurations, which are depicted in Fig. 1. It is assumed that each configuration consists of $N_{t}$ identical radiating antenna

\footnotetext{
${ }^{1}$ Note that we assume that the noise power is equal to 1 , such that $\rho$ represents the average SNR and is therefore dimensionless.
}

element, which are uniformly positioned in a plane. For the URPA configuration, the total number of antenna elements is $N_{t}=N_{x} \times N_{y}$. For the UHPA and UCPA configurations, and if the number of antenna elements on the $m$-th hexagon ring and circle, respectively, is $6 \mathrm{~m}$, the total number of antenna elements can be calculated as

$$
N_{t}=1+\sum_{m=1}^{M} 6 m
$$

where $M$ denotes the number of rings/circles for a $\mathrm{UH}$ PA/UCPA configuration. It is assumed that the inter-elements spacing at the horizontal direction is $d=\lambda / 2$ and the number of antenna elements equals to 90 , the geometric area of URPA, UHPA and UCPA is $22.5 \lambda^{2}, 16.24 \lambda^{2}$ and $19.64 \lambda^{2}$, respectively. We now present the steering vector and array factor of three antenna arrays as follows:

1) URPA Analysis: A URPA configuration, which is depicted in Fig. 1(a), consists of $N_{x}$ antennas in each row (each row is a ULA) and $N_{y}$ antennas in each column (each column is also a ULA) in the horizontal and vertical directions, respectively, and the corresponding inter-element spacing is denoted as $d_{x}$ and $d_{y}$ respectively. Note that $N_{x}$ and $N_{y}$ are arbitrary integers. In the far-field regime, the steering vector for the URPA configuration is represented by [12]

$$
\mathbf{a}(\phi, \theta)=\mathbf{v}_{x}(\phi, \theta) \otimes \mathbf{v}_{y}(\phi, \theta),
$$

where $\mathbf{v}_{x}(\cdot)$ and $\mathbf{v}_{y}(\cdot)$ can be viewed as the steering vectors on the horizontal and vertical direction, respectively, with

$$
\mathbf{v}_{x}(\phi, \theta)=\left[\begin{array}{llll}
1 & e^{j k d_{x} \sin \theta \cos \phi} & \cdots & e^{j k\left(N_{x}-1\right) k d_{x} \sin \theta \cos \phi}
\end{array}\right]^{T}
$$

and

$$
\mathbf{v}_{y}(\phi, \theta)=\left[\begin{array}{llll}
1 e^{j k d_{y} \sin \theta \sin \phi} & \cdots & e^{j k\left(N_{y}-1\right) k d_{y} \sin \theta \sin \phi}
\end{array}\right]^{T},
$$

where $k=2 \pi / \lambda$ is the wavenumber and $\lambda$ is the carrier wavelength. In order to adjust beam of antenna array, we need to multiply by the weight vector. According to (5), the array factor for URPA can be calculated as

$$
A F_{\mathrm{R}}(\phi, \theta)=\sum_{m=1 n=1}^{N_{x}} \sum_{m n}^{N_{y}} w_{m} e^{j\left[(m-1) k d_{x} \sin \theta \cos \phi+(n-1) k d_{y} \sin \theta \sin \phi\right]},
$$

where $w_{m n}$ denote the weight vector (i.e., antenna elements excitation), which is designed to steer the planar antenna array towards the desired direction.

2) UHPA Analysis: A UHPA configuration, which is depicted in Fig. 1(b), consists of $M$ hexagon rings, the length of every hexagon ring side is $d_{m}$, the number of antenna element on the $m$-th hexagon ring is $6 m$ while the antenna elements are uniformly distributed in the hexagonal side, and inter-element spacing on the horizontal and vertical direction is set to $d_{x}$ and $d_{h}$, respectively. Then, in the far-field regime, the channel response vector for the UHPA configuration can be given by [13, Page 307]

$$
\mathbf{a}(\phi, \theta)=\left[\begin{array}{lllllll}
\mathbf{v}_{M} & \ldots & \mathbf{v}_{1} & \mathbf{v}_{0} & \mathbf{v}_{-1} & \ldots & \mathbf{v}_{-M}
\end{array}\right]^{T} .
$$


where $\mathbf{v}_{n}$ (i.e. $n=-M, \ldots, M$ ) denotes the different vectors at a row (each row is a ULA), which depends on the subscript $n$. When the subscript $n$ is an even number, the vector $\mathbf{v}_{n}$ can be given by

$\mathbf{v}_{n}=\delta\left[e^{-j\left(M-\frac{n}{2}\right) k d_{x} v} \cdots e^{-j k d_{x} v} 1 e^{j k d_{x} v} \cdots e^{j\left(M-\frac{n}{2}\right) k d_{x} v}\right]$,

where $\delta=e^{-j n k d_{h} v}$ with $d_{h}=\sqrt{3} d_{x} / 2$ and $v=\sin \theta \cos (\phi)$. When the subscript $n$ is an odd number, the vector $\mathbf{a}_{n}$ is given by

$$
\mathbf{v}_{n}=\delta\left[e^{-j\left(M-\frac{n-1}{2}\right) k d_{x} v} \cdots e^{-j k d_{x} v} e^{j k d_{x} v} \cdots e^{j\left(M-\frac{n-1}{2}\right) k d_{x} v}\right],
$$

According to (9), the array factor for UHPA is calculated as

$$
\begin{aligned}
A F_{\mathrm{H}}(\phi, \theta)= & \sum_{m=-M}^{M} e^{j \pi\left[m \sin \theta \sin \phi-\frac{2 M-|m|}{2} \sin \theta \cos \phi-\frac{m}{2} \sin \theta \cos \phi\right]} \\
& \sum_{n=0}^{2 M-|m|} w_{m n} e^{j \pi n \sin \theta \cos \phi},
\end{aligned}
$$

3) UCPA Analysis: The structure of a UCPA is similarly to a UHPA, as showed in Fig. 1(c), with the hexagon ring replaced to circle ring. In the UCPA configuration, in which $6 m$ antenna elements are uniformly placed around the circular edge of the $m$-th radius. Then, in the far-field regime, the steering vector of a UCPA configuration is expressed as

$$
\mathbf{a}(\phi, \theta)=\left[1 e^{j k r_{m} \sin \theta \cos \left(\phi-\bar{\phi}_{1}\right)} \cdots e^{j k r_{m} \sin \theta \cos \left(\phi-\bar{\phi}_{m n}\right)}\right]^{T},
$$

where $m$ denotes the number of circle ring, $r_{m}$ denotes the radius of the circular array, $\bar{\phi}_{m n}$ denotes the angle between the $n$-th antenna elements on the $m$-th circle ring and the $x$-direction. According to (13), the array factor for a UCPA configuration can be calculated as

$$
A F_{\mathrm{C}}(\phi, \theta)=1+\sum_{m=1}^{M} \sum_{n=1}^{6 m} w_{m n} e^{-j\left(\pi m \sin \theta \cos \left(\phi-\frac{\pi n}{3 m}\right)\right)} .
$$

So far, the steering vectors and array factors for the proposed planar antenna arrays have been derived. It is shown that the steering vector and array factor mainly depend on the number of antenna elements, the array configuration and the antenna elements excitation (i.e., the weight vector). Based on these expressions, the antenna array directivity/gain and the achievable SE will be investigated in the following section.

\section{Performance Analysis}

There are many radiation pattern characteristics for antenna arrays, such as antenna array directivity/gain, beamwidth, main lobe, and effective aperture, etc. In this section, we focus on the antenna array directivity/gain calculation and then, on the achievable SE estimation in order to check the effect of different planar antenna arrays configurations on mmWave massive MIMO systems.

In general, the radiation field form of each of the antenna elements can be given by [11]

$$
E_{m}(r, \phi, \theta)=A f(\phi, \theta) \frac{e^{-j k r}}{r},
$$

where $A$ is the nominal field amplitude and $f(\phi, \theta)$ is the normalized to unity electric field pattern, and $r$ is the distance between the element and the reference point. According to the pattern multiplication principle [11], the antenna array total electrical field of the antenna array is,

$$
E=E_{m} A F(\phi, \theta) .
$$

The antenna directivity is defined as

$$
D\left(\phi_{T}, \theta_{T}\right)=4 \pi \frac{U}{P_{\mathrm{rad}}},
$$

where

$$
P_{\mathrm{rad}}=\int_{\phi=0}^{2 \pi} \int_{\theta=0}^{\pi} U \sin \theta d \theta d \phi,
$$

is the total radiated power, and $U=|E|^{2} r^{2} / 2 \eta_{0}$ is the radiation intensity with $\eta_{0}$ being the intrinsic impedance of the medium. Substituting (15) into (16) and combining it with $U=|E|^{2} r^{2} / 2 \eta_{0}$, we obtain

$$
U=\frac{|A|^{2}}{2 \eta_{0}}|f(\phi, \theta) A F(\phi, \theta)|^{2} .
$$

Substituting (18) and (19) into (17), the array directivity is given by

$$
D(\phi, \theta)=\frac{4 \pi|f(\phi, \theta) A F(\phi, \theta)|^{2}}{\int_{\phi=0}^{2 \pi} \int_{\theta=0}^{\pi}|f(\phi, \theta) A F(\phi, \theta)|^{2} \sin \theta d \theta d \phi} .
$$

In our calculations, we assume that each antenna array consists of isotropic elements, and thus $f(\phi, \theta)=1$ [13, Page 60]. Consequently, the directivity of the antenna array is determined by the array factor. Moreover, assuming that the antenna arrays are lossless or equivalently have unity efficiency, directivity is equal to antenna gain. Hence

$$
G(\phi, \theta)=D(\phi, \theta)=\frac{4 \pi|A F(\phi, \theta)|^{2}}{\int_{\phi=0}^{2 \pi} \int_{\theta=0}^{\pi}|A F(\phi, \theta)|^{2} \sin \theta d \theta d \phi} .
$$

From (21), it is obvious that the antenna array gain is proportional to the squared absolute value of the antenna factor, as it was previously mentioned in (6). In (21), the double integral was estimated via numerical technique. For the URPA, UHPA and UCPA the result was 772.977, 768.463 and 786.239, respectively. Hence, the maximum gain was $42.39 \mathrm{~dB}, 42.63$ $\mathrm{dB}$ and $43.00 \mathrm{~dB}$, respectively at $(\phi, \theta)=(0,0)$.

We now study the effect of different antenna array configurations on the achievable rate. We assume that equal power allocation among antenna elements is adopted and the transmitter has perfect channel state information. From (1), under the assumption of Gaussian input signaling, the achievable $\mathrm{SE}$ of the system is expressed as

$$
R=\mathbb{E}\left\{\log _{2} \operatorname{det}\left(\mathbf{I}_{N_{r}}+\frac{\rho}{N_{t}} \mathbf{H}^{H} \mathbf{H}\right)\right\},
$$


From (22), it is worth noting that the expectation is taken over all channel realizations of $\mathbf{H}$ and the channel is needed to be ergodic. We observe that it is difficult to derive closedform expressions on the achievable SE for hybrid architecture. Alternatively, we seek to tractable bounds on the total achievable SE of hybrid architecture massive MIMO system, which enable us to draw engineering insights into the performance of system. With the aid of the Hadamard inequality property, an upper bound on the achievable SE can be calculated as

$$
R \leq \mathbb{E}\left\{\log _{2}\left(\prod_{r=1}^{N_{r}}\left[\mathbf{I}+\frac{\rho}{N_{t}} \mathbf{H}^{H} \mathbf{H}\right]_{r r}\right)\right\},
$$

By applying Jensen's inequality, the upper bound can be further calculated as

$$
R \leq \sum_{r=1}^{N_{r}} \log _{2}\left(1+\frac{\rho}{N_{t}} \mathbb{E}\left\{\left\|\mathbf{h}_{r}\right\|^{2}\right\}\right),
$$

where $\mathbf{h}_{r}$ denotes the $r$-th row of $\mathbf{H}$. This means that given a constraint on $\left\|\mathbf{h}_{r}\right\|^{2}$, the achievable SE can be maximum. According to (2), we know that the variance of $a_{p l}$ equals to 1 and the antenna array gain is set as one within the range of AoDs/AoAs. Therefore, we have

$$
\mathbb{E}\left\{\left\|\mathbf{h}_{r}\right\|^{2}\right\}=\mathbb{E}\left\{\left|a_{p l}\right|^{2}\right\} \Lambda_{t}^{2} \Lambda_{r}^{2} N_{t} \leq \Lambda_{t}^{2} \Lambda_{r}^{2} N_{t} .
$$

Substituting this result into (25), we obtain

$$
R_{\text {upper }}=N_{r} \log _{2}(1+\rho) .
$$

From (26), we notice that the upper bound on the achievable SE depends on the antenna array gain of transmitter and receiver, and the transmit power. Generally speaking, the antenna array gain increases with the number of antenna elements. This result implies the upper bound infinitely large as the number of transmitter antenna grows without bound. In addition, it can be clearly seen that the achievable SE has no relative with the number of antennas at the transmitter and the proposed antenna array cases, only depends on the number of antennas at the receiver the and the SNR.

\section{Simulation Results}

In this section, we provide simulation results to compare the proposed antenna array beam pattern and present the total achievable SE of the system for the proposed planar antenna arrays. In simulations, we assume that the receiver is deployed ULA configuration and the transmitter is equipped with the URPA/UHPA/UCPA configuration, the height of the transmitter is fixed to $h=50 \mathrm{~m}$, the radius of circular-shaped cell is set to $r=500 \mathrm{~m}$ and the radius of guard zone is set to $r_{0}=50 \mathrm{~m}$, the spread azimuths at the transmitter are set to $\Delta \theta^{t}=10.2^{\circ}$ and $\Delta \phi^{t}=0^{\circ}$, respectively; the spread azimuths at the receiver are set to $\Delta \theta^{r}=15.6^{\circ}$ and $\Delta \phi^{r}=$ $6^{\circ}$, respectively, the inner antenna spacing is $d=\lambda / 2$. These parameters are obtained from the measurement results of [7], while the channel model is generated as described in (2).

In Fig. 2 from (a) to (c), we depict the normalized antenna array gain in spherical coordinates. Due to the $\theta=\pi / 2$ plane symmetry, only the range $\theta \in[-\pi / 2 \pi / 2]$ is depicted. In our

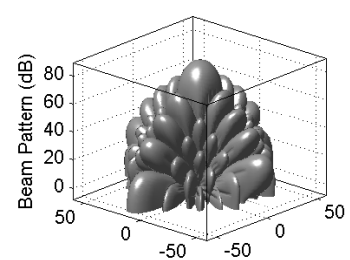

(a)

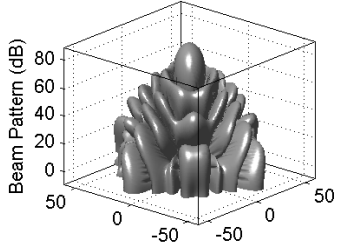

(b)

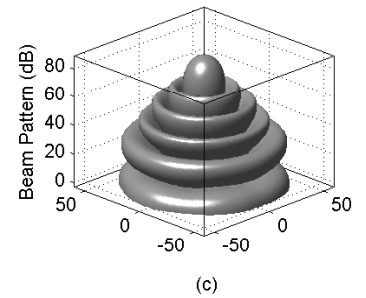

Fig. 2. Antenna array gain in spherical coordinates for the proposed planner antenna arrays. (a) The URPA configuration (b) The UHPA configuration (c) The UCPA configuration.

simulation, the number of antenna elements for URPA (UHPA and UCPA) is set to $N_{t}=90$ (91), the inter-element spacing is set to $d_{x}=d_{y}=\lambda / 2\left(d_{h}=\sqrt{3} d_{y} / 2\right)$, the weights factor is set to $w_{m n}=1$ and the desired direction is set to $(\phi, \theta)=$ $\left(0^{\circ}, 0^{\circ}\right)$. It is evident that all three antenna arrays has their 3D beam pattern and are different directional with the main lobe located at $(\phi, \theta)=\left(0^{\circ}, 0^{\circ}\right)$ and $(\phi, \theta)=\left(0^{\circ}, \pi\right)$. Additionally, the UCPA has almost omni-directional radiation pattern in the horizontal plane (i.e., $\theta=\pi / 2$ ), however the maximum gain in this plane has level $50 \mathrm{~dB}$ from the maximum lobe.

Fig. 3 depicts the normalized antenna array gain in the $\theta=\pi / 2$ plane for $\phi \in[-\pi / 2 \pi / 2]$. The beamwidth and especially the half power beam width (HPBW) for all the three antenna arrays is almost identical: $11.30^{\circ}, 10.15^{\circ}$, and $10.82^{\circ}$, respectively. Additionally, the first null for all cases occurs around $13.00^{\circ}$. However, it is evident that the antenna arrays have different side lobes. Specifically, the URPA, UHPA and UCPA have sidelobe level $-12.90 \mathrm{~dB},-18.86 \mathrm{~dB}$ and $-17.65 \mathrm{~dB}$, respectively, we know that UHPA configuration is the lowest side lobe level compared to the URPA and UCPA configurations. This indicate that adopting UHPA configuration yields severe interference.

In Fig. 4, the simulated total achievable SE for the proposed planer antenna array configurations are plotted against the SNR. As we can see, the total achievable SE of the proposed antenna array configurations is also identical (the URPA configurations experiences a little degradation). This indicates that antenna array configurations have identical effect on the performance of massive MIMO systems. We see that the upper bound is consistently above the simulation result in the entire SNR regime, which is in accordance with the result in (25). In addition, we also see that the gap on the achievable SE between the upper bound and simulation results increases with the SNR. Especially, the gap becomes relatively obvious when $\mathrm{SNR}=5 \mathrm{~dB}$. This because the transmitter power plays a 


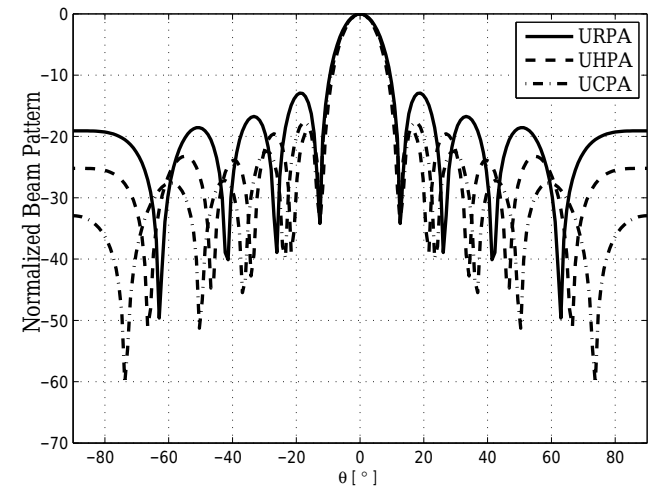

Fig. 3. Normalized Beam Pattern for the proposed planner antenna arrays.

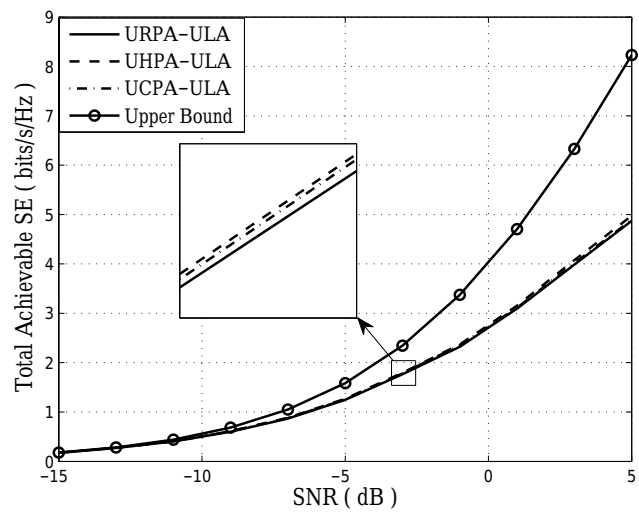

Fig. 4. The total achievable SE for the proposed antenna array configurations at the transmitter and a ULA configuration at the receiver.

dominant role and the channel gain is negligible.

TABLE I

PHYSICAL AND RADIATION CHARACTERISTICS

\begin{tabular}{cccc}
\hline Metric & URPA & UHPA & UCPA \\
\hline Antenna number & 90 & 91 & 91 \\
Inter-element spacing & $\lambda / 2$ & $\lambda / 2$ & $\lambda / 2$ \\
Maximum gain (dB) & 42.39 & 42.63 & 43.00 \\
HPBW (3 dB) & $11.30^{\circ}$ & $10.15^{\circ}$ & $10.82^{\circ}$ \\
Side lobe level (dB) & -12.90 & -18.86 & -17.65 \\
Geometric area & $22.5 \lambda^{2}$ & $16.24 \lambda^{2}$ & $19.64 \lambda^{2}$ \\
\hline
\end{tabular}

Based on the above analysis, we summarize the physical and radiation characteristics of the proposed antenna arrays in Table II. It is clear that the three antenna configurations have not only almost identical maximum gain, but also identical HPBW. Equivalently, these radiation characteristics (i.e., maximum gain and HPBW) have the most significant effect on the achievable sum rate. However, the UHPA has the highest side lobe level compared to the URPA and UCPA, but has the smaller geometric area. Therefore, when first priority is the compact size, the UHPA configuration is a valid option. From the above discussion, we conclude that 2D antenna array has itself metric, it is hard to exact evaluate which type of the antenna array configuration is more suitable for massive MIMO systems and it needs to concern practical issues.

\section{CONCLUSION}

In this paper, we investigated the performance of three different planar arrays, namely URPA, UCPA, or UHPA, and provided the simulated achievable sum rate. An important observation is that the considered 2D arrays had almost identical performance and yielded the same achievable SE for a clustered mmWave channel model. We conclude that the 2D planar antenna array configurations almost have no effect on the performance of massive MIMO systems and our results provide insight on design criteria of the 2D planer antenna array, which will be most useful in the practical application of mmWave massive MIMO systems.

\section{ACKNOWLEDGMENT}

This work was supported in part by the National Science Foundation (NSFC) for Distinguished Young Scholars of China with Grant 61625106, the National Natural Science Foundation of China under Grants 61531011, 61571112, a Foundation for the Author of National Excellent Doctoral Dissertation of P. R. China (FANEDD) under Grant 201446, and the International Science \& Technology Cooperation Program of China under Grant 2014DFT10300.

\section{REFERENCES}

[1] T. L. Marzetta, "Noncooperative cellular wireless with unlimited numbers of base station antennas," IEEE Trans. Wireless Commun., vol. 9, no. 11, pp. 3590-3600, Nov. 2010.

[2] L. Lu, G. Y. Li, A. L. Swindlehurst, A. Ashikhmin, and R. Zhang, "An overview of massive MIMO: Benefits and challenges," IEEE J. Sel. Topics Signal Processing, vol. 14, no. 15, pp. 136-146, Oct. 2014.

[3] Z. Pi and F. Khan, "A millimeter-wave massive MIMO system for next generation mobile broadband," in Proc. IEEE ASILOMAR, Jun. 2012, pp. 1010-1017.

[4] Y. H. Nam, B. L. Ng, K. Sayana, Y. Li, J. Zhang, Y. Kim, and J. Lee, "Full-dimension MIMO (FD-MIMO) for next generation cellular technology," IEEE Commun. Mag., vol. 51, no. 6, pp. 172-186, Jun. 2013.

[5] W. Tan, S. Jin, J. Wang, and Y. Huang, "Achievable sum-rate analysis for massive MIMO systems with different array configurations," in Proc. IEEE WCNC, Mar. 2015, pp. 61-66.

[6] A. Forenza, D. Love, and R. W. Heath, Jr., "Simplified spatial correlation models for clustered MIMO channels with different array configurations," IEEE Trans. Veh. Technol., vol. 56, no. 4, pp. 1924-1934, Jun. 2007.

[7] M. R. Akdeniz, Y. Liu, M. K. Samimi, S. Rangan, T. S. Rappaport, et al., "Millimeter wave channel modeling and cellular capacity evaluation," IEEE J. Sel. Areas Comm., vol. 32, no. 6, pp. 1164-1179, Apr. 2014.

[8] M. R. Akdeniz, Y. Liu, M. K. Samimi, S. Sun, S. Rangan, T. S. Rappaport, and E. Erkip. "Synthesizing omnidirectional antenna patterns, received power and path loss from directional antennas for $5 \mathrm{G}$ millimeter-wave communications," Avaiable. [Online], arXiv:1511.07271, 2015.

[9] O. E. Ayach, S Rajagopal, S. A. Surra, Z. Pi and R. W. Heath, Jr. "Spatially sparse precoding in millimeter wave MIMO systems," IEEE Trans. Wireless Commun., vol. 13, no. 3, pp. 1499-1513, Apr. 2014.

[10] T. S. Rappaport, E. B. Dor, J. N. Murdock, and Y. Qiao, " $38 \mathrm{GHz}$ and $60 \mathrm{GHz}$ angle-dependent propagation for cellular \& peer-to-peer wireless communications," in Proc. IEEE ICC, Jun. 2012, pp. 104-110.

[11] C. A. Balanis, Antenna theory: Analysis and design. John Wiley Sons, 2012.

[12] J. H. Chen, "When does asymptotic orthogonality exist for very large arrays?" in Proc. IEEE GLOBECOM, Nov. 2013, pp. 6-10.

[13] L. Harry and V. Trees, Optimum array processing: Detection, estimation, and modulation theory. New York: John Wiler Sons, 2002.

[14] W. Lee, S. R. Lee, H. B. Kong, and I. Lee, "3D beamforming designs for single user MISO systems," in Proc. IEEE GLOBECOM., Jun. 2013, pp. 3914-3919. 